\title{
Biological Modification of the Fatty Acid Group in an Emulsan by Supplementing Fatty Acids under Conditions Inhibiting Fatty Acid Biosynthesis
}

\author{
PIL KIM, ${ }^{1}$ DEOK-KUN OH, ${ }^{2}$ JUNG-KUL LEE, ${ }^{3}$ SANG-YONG KIM, ${ }^{3}$ AND JUNG-HOE KIM ${ }^{1}$ * \\ Department of Biological Sciences, Korea Advanced Institute of Science and Technology, Taejon 305-701, ${ }^{1}$ \\ Department of Bioscience and Biotechnology, Sejong University, Seoul 143-747, ${ }^{2}$ \\ and BioNgene Co., Kyunggi-Do 445-930, ${ }^{3}$ Korea
}

Reccived 28 February 2000/Acccptcd 19 June 2000

\begin{abstract}
When the concentration of the antibiotic cerulenin was increased up to $3.0 \mathrm{mg} / l$ in medium containing ethanol as a carbon source, the specific growth rate of Acinetobacter calcoaceticus and the fatty acid content of the emulsan decreased from $0.179 \mathrm{~h}^{-1}$ and $13.9 \%$ to $0.015 \mathrm{~h}^{-1}$ and $3.4 \%$, respectively. The emulsifying activity in medium containing cerulenin decreased with increasing cerulenin concentration. In the culture containing $3.0 \mathrm{mg} / l$ cerulenin, fatty acid biosynthesis was inhibited. Various fatty acids were added to this inhibitory culture as a second carbon source to modify the fatty acid group in the emulsan. When an odd-numbered fatty acid was added, the resulting emulsan was found to have other odd-numbered fatty acids that were not present originally. Among the emulsan produced from even-numbered fatty acids, the emulsan produced from myristic acid (C14) contained the greatest amount of the same-numbered fatty acids. When the amount of supplemental myristic acid was increased, the myristic acid content in the emulsan increased, but its emulsifying activity decreased.
\end{abstract}

[Key words: emulsan, biological modification, fatty acid, cerulenin]

Microbial biosurfactants have recently been studied intensively because of their useful functional properties, such as emulsification, phase separation, wetting, foaming, solubilization, corrosion inhibition, and viscosity reduction. Biosurfactants have marked advantages over chemically synthesized surface-active compounds and can replace conventional surfactants in many areas including agriculture, industrial cleaning, the food and beverage, textile, cosmetics, and pharmaceutical industries. Their advantages are numerous: they have many potential applications due to their novel structural characteristics and physical properties; they are produced from renewable substrates; they can be readily modified by genetic engineering, biologically or biochemically; and they are biodegradable (1).

Emulsan, one of the most promising biosurfactants, is an extracellular lipopolysaccharide biosurfactant produced by Acinetobacter calcoaceticus RAG-1. Emulsan has an average molecular weight of about $1000 \mathrm{kDa}$ and can be produced from water-immiscible substrates, as well as water-soluble substrates such as ethanol (2). Emulsan consists of a hydrophilic carbohydrate backbone and a group of hydrophobic fatty acids linked by an ester or amide, which gives emulsan its amphiphilic properties (3). The carbohydrate backbone of emulsan consists of D-galactosamine, D-galactosaminuronic acid, and an unidentified monosaccharide. In addition, emulsan contains $15-25 \%$ protein with an average molecular weight of $10-90 \mathrm{kDa}(4,5)$. The protein can be removed leaving apoemulsan which retains the properties of the native emulsan (6).

Belsky et al. (3) suggested that both the total amount and distribution of fatty acids in an emulsifier play an important role in its emulsifying activities. The fraction

\footnotetext{
* Corresponding author.
}

of 3-hydroxymyristic acid residues in lichenicin A, a lipopeptide biosurfactant from Bacillus licheniformis BAS50, was reported to be one of the most important factors influencing its surface activity (7). We previously reported that the 3-hydroxydodecanoic acid content of cmulsan playcd a role in its emulsifying activity (8). It was also reported that modifying the fatty acid in biosurfactants affected the properties of each biosurfactant $(9,10)$. By using different strains and cultivation conditions, the fatty acid group in biosurfactants can be modified to improve their properties (11).

In this study, we attempted to modify emulsan biologically by supplementing the media with fatty acids under conditions inhibiting fatty acid biosynthesis, to change its functional properties.

\section{MATERIALS AND METHODS}

Microorganism and culture conditions $A$. calcoaceticus RAG-1 ATCC 31012 was used to prepare the emulsan products. The basal medium contains $5 \mathrm{ml}$ ethanol, $2 \mathrm{~g}$ urea, $16.9 \mathrm{~g} \mathrm{~K}_{2} \mathrm{HPO}_{4}, 7.3 \mathrm{~g} \mathrm{K \textrm {K } _ { 2 }} \mathrm{PO}_{4}, 0.5 \mathrm{~g}$ $\mathrm{MgSO}_{4} \cdot 7 \mathrm{H}_{2} \mathrm{O}, 1.08 \mathrm{mg} \mathrm{CaCl} \cdot 2 \mathrm{H}_{2} \mathrm{O}, 1.87 \mathrm{mg} \mathrm{CuSO}{ }_{4} \cdot$ $5 \mathrm{H}_{2} \mathrm{O}, 1.81 \mathrm{mg} \mathrm{FeSO}_{4} \cdot 7 \mathrm{H}_{2} \mathrm{O}, 1.78 \mathrm{mg} \mathrm{MnSO}_{4} \cdot 4 \mathrm{H}_{2} \mathrm{O}$, $1.27 \mathrm{mg} \mathrm{ZnSO}_{4} \cdot 7 \mathrm{H}_{2} \mathrm{O}, 2.36 \mathrm{mg} \mathrm{CoCl}_{2} \cdot 6 \mathrm{H}_{2} \mathrm{O}$, and 2.10 mg $\mathrm{Na}_{2} \mathrm{MoO}_{4}$ per liter (12). The mineral solutions were sterilized separately to avoid precipitation. The medium used to biologically modify emulsan under conditions inhibiting fatty acid biosynthesis consisted of $0.5 \%(\mathrm{v} / \mathrm{v})$ ethanol, $0.5 \%(\mathrm{v} / \mathrm{v})$ fatty acids, which were added after $24 \mathrm{~h}$ cultivation, and basal medium containing $3.0 \mathrm{mg} / \mathrm{l}$ cerulenin instead of $0.5 \%$ ethanol. The frozen culture was thawed in a water bath at $30^{\circ} \mathrm{C}$ and inoculated into a $250-\mathrm{ml}$ Erlenmeyer flask containing $50 \mathrm{ml}$ of the basal mcdium. The flask was incubated in a rotary-shaking incubator (NBS, USA) at $30^{\circ} \mathrm{C}, 250 \mathrm{rpm}$, for $24 \mathrm{~h}$, and cul- 
tivated for $16 \mathrm{~h}$ in fresh medium. Thereafter, $5 \mathrm{ml}$ of the subculture broth was transferred to a 2.8- $l$ Furnback flask containing $500 \mathrm{ml}$ of either the basal medium or the medium for biological modification. Fermentation experiments were performed in a rotary-shaking incubator at $30^{\circ} \mathrm{C}, 250 \mathrm{rpm}$, for $72 \mathrm{~h}$. The initial $\mathrm{pH}$ of the culture was in the range of 7.0 to 7.2 .

Isolation and purification of emulsan Cells were removed from the culture broth by centrifugation. To precipitate the lipopolysaccharide, $47 \%$ ammonium sulfate was added to the supernatant solution and the mixture was allowed to stand overnight at $4{ }^{\circ} \mathrm{C}$. The precipitate was centrifuged, dialyzed using dialysis tubing (molecular weight cut off 12-14 kDa) against distilled water in the cold room, and freeze-dried. The emulsan was extracted from the freeze-dried sample for $1 \mathrm{~d}$ with diethyl ether using a Soxhlet apparatus and then dried. The emulsan sample obtained was used for structural analysis (4).

Analytical methods The fatty acids in emulsan were determined using Belsky's method (2). Purified emulsan, $5 \mathrm{mg}$, was hydrolyzed for $16 \mathrm{~h}$ in $2 \mathrm{ml}$ of $6 \mathrm{~N}$ $\mathrm{HCl}$ at $100^{\circ} \mathrm{C}$ while purging with nitrogen, then cooled and extracted thrice with diethyl ether. The sample was dried by evaporating off the diethyl ether and analyzed by GLC-FID using a capillary column. The oven temperature was increased from $100^{\circ} \mathrm{C}$ to $280^{\circ} \mathrm{C}$ at a rate of $5^{\circ} \mathrm{C} / \mathrm{min}$ and benzoic acid was used as an internal standard. Each peak was determined by using GC-MS.

The emulsifying activity of emulsan was measured using a modification of Zuckerberg's method (4). A 1mg emulsan sample was dissolved in $0.1 \mathrm{ml}$ of test oil (hexadecane : 2-methylnaphthalene $=1: 1, \mathrm{v} / \mathrm{v}$ ) in a 125$\mathrm{ml}$ Erlenmeyer flask containing $7.5 \mathrm{ml}$ of TM buffer $(20$ $\mathrm{mM}$ Tris- $\mathrm{HCl}, \mathrm{pH} 7.2,10 \mathrm{mM} \mathrm{MgSO} 4 \cdot 7 \mathrm{H}_{2} \mathrm{O}$ ). The flask was reciprocally shaken at $30^{\circ} \mathrm{C}$ for $1 \mathrm{~h}$ (150 strokes/ $\mathrm{min}$ ) and the buffer solution was used for the activity measurement. The emulsifying activity was determined by measuring the increase in the optical density at 600 $\mathrm{nm}$. One unit of emulsifying activity was defined as one absorbance unit using a spectrophotometer.

The hydrophilic-lipophilic balance of emulsan was determined using the back-titration method according to Greenwald et al. (13). A $0.01 \mathrm{~g}$ emulsan sample was dissolved in $10 \mathrm{ml}$ of distilled water and titrated using an oil mixture ( $4 \%$ benzene $+96 \%$ 1,4-dioxane) until the first trace of turbidity appeared. The value of the hydrophilic-lipophilic balance was determined from the values for the sucrose-lipid esters (Ryoto esters) used as standards. The intrinsic viscosity was measured using a capillary viscometer in a $25^{\circ} \mathrm{C}$ water bath. The specific growth rate was determined from the slope of the logarithm of optical density $(600 \mathrm{~nm})$ versus time curve.

\section{RESULTS}

Effect of cerulenin concentration on the growth of $A$. calcoaceticus and the biosynthesis of fatty acids in emulsan Cerulenin, (2S) (3R) 2,3-epoxy-4-oxo-7,10-dodecadienoylamide, is an anti-lipogenic antibiotic produced in Cephalosporium caerulens $(14,15)$. Cerulenin, which has a broad effective spectrum range against bacteria and fungi, inhibits the condensation reaction in fatty acid biosynthesis (16). To determine the inhibitory conditions, the effect of cerulenin concentration on the specific growth rate of $A$. calcoaceticus RAG-1 was investi-

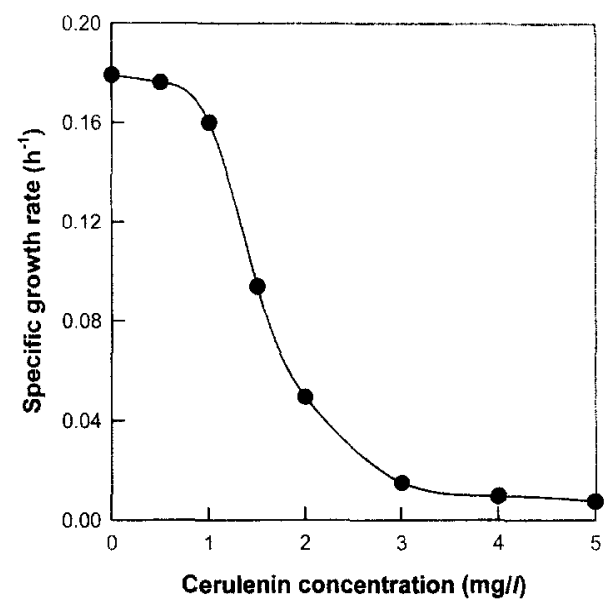

FIG. 1. Effect of cerulenin concentration on the specific growth rate of $A$. calcoaceticus.

gated in the basal medium. When the cerulenin concentration was increased, the specific growth rate decreased and was almost completely inhibited at cerulenin concentrations above $3.0 \mathrm{mg} / l$, as shown in Fig. 1. Therefore, a culture containing $3.0 \mathrm{mg} / \mathrm{l}$ cerulenin was defined as one in which fatty acid biosynthesis was inhibited.

The properties of emulsan produced in the presence of various cerulenin concentrations are shown in Table 1. As the cerulenin concentration increased, the hydrophilic-lipophilic balance and intrinsic viscosity increased. These results suggest that the emulsan produced at a higher cerulenin concentration became more hydrophilic and had a larger hydrodynamic volume. The emulsifying activity was higher in the presence than in the absence of cerulenin, although the activity decreased with increasing cerulenin concentration in medium containing cerulenin.

Figure 2 shows the effect of cerulenin concentration on the fatty acid content in medium containing $1.0 \%$ ethanol. The fatty acid content in the emulsan decreased from $13.9 \%$ to $3.4 \%$, as the cerulenin concentration was increased from 0.0 to $3.0 \mathrm{mg} / l$. The relationship between the fatty acid content $(Y)$ and the cerulenin concentration $(\mathrm{X})$ is given by $\mathrm{Y}-13.36-3.34 \mathrm{X}\left(\mathrm{r}^{2}-0.983\right)$. The slope of this line represents the degree of inhibition of fatty acid biosynthesis by cerulenin, and shows that a 1 $\mathrm{mg} / \mathrm{l}$ increase in cerulenin decreased the fatty acid content by $3.34 \%$.

Biological modification of emulsan by addition of odd-numbered fatty acids under conditions inhibiting fatty acid biosynthesis To modify the fatty acid composition of emulsan, odd-numbered fatty acids were added to cultures grown for $24 \mathrm{~h}$ under the conditions inhibiting fatty acid biosynthesis. We determined whether the fatty acid added under these conditions was directly

TABLE 1. Properties of emulsan produced in the presence of various cerulenin concentration

\begin{tabular}{lccccc}
\hline $\begin{array}{l}\text { Cerulenin } \\
\text { concentration }(\mathrm{mg} / l)\end{array}$ & 0.0 & 1.0 & 1.5 & 2.0 & 3.0 \\
\hline $\begin{array}{c}\text { Emulsifying activity } \\
(\mathrm{U} / \mathrm{mg})\end{array}$ & 1.50 & 2.44 & 2.42 & 2.23 & 1.70 \\
$\begin{array}{c}\text { Hydrophilic-lipophilic } \\
\text { balance } \\
\begin{array}{c}\text { Intrinsic viscosity } \\
(\mathrm{dl} / \mathrm{g})\end{array}\end{array}$ & 8.2 & 11.6 & 13.9 & 15.3 & 17.2 \\
\hline
\end{tabular}




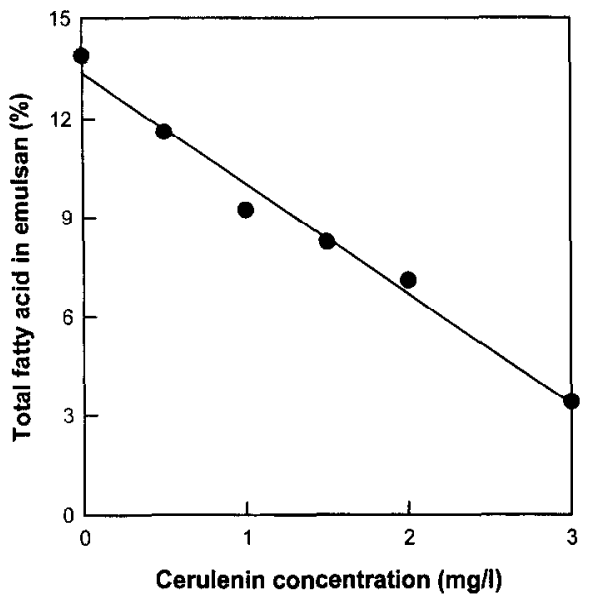

FIG. 2. Effect of cerulenin concentration on the total fatty acid content.

incorporated into emulsan. The odd-numbered fatty acids tested were heptanoic acid (C7), undecanoic acid (C11), tridecanoic acid (C13), pentadecanoic acid (C15), heptadecanoic acid (C17), and nonadecanoic acid (C19). The control consisted of the emulsan produced from $0.5 \%$ ethanol without fatty acid supplementation under the conditions inhibiting fatty acid biosynthesis (Table 2).

The changes in the fatty acid composition that occurred with odd-numbered supplemental fatty acids were investigated. Emulsan produced without fatty acid supplementation contained a high proportion of long-chain fatty acids, such as $\mathrm{C} 18$ and $\mathrm{C} 20$, whereas the emulsan produced with odd-numbered supplemental fatty acids had a high proportion of short-chain fatty acids, such as $\mathrm{C} 8, \mathrm{C10}$, and $\mathrm{C} 12$. All the emulsan samples produced with odd-numbered supplemental fatty acids contained trace amounts of odd-numbered fatty acids, although not the same odd-numbered acid. The trace amounts of odd-numbered fatty acids found included $\mathrm{C}$, $\mathrm{C} 11, \mathrm{C} 13$, $\mathrm{C} 15$, and $\mathrm{C} 17$, and this was confirmed by MS fragmenta- tion. The emulsifying activity, however, was not related to supplementation with odd-numbered fatty acids (data not shown).

Biological modification of emulsan by addition of even-numbered fatty acids under conditions inhibiting fatty acid biosynthesis To modify the fatty acid composition of emulsan, $0.5 \%$ of even-numbered fatty acids were added to cultures grown for $24 \mathrm{~h}$ under the conditions inhibiting fatty acid biosynthesis. The even-numbered fatty acids tested were caprylic acid (C8), capric acid (C10), lauric acid (C12), myristic acid (C14), palmitic acid $(\mathrm{C} 16)$, and stearic acid $(\mathrm{C} 18)$. The changes in the fatty acid composition of emulsan produced by even-numbered supplemental fatty acids were similar to those produced by odd-numbered supplemental fatty acids (Table 3). Of the emulsan produced from evennumbered fatty acids, that produced from myristic acid (C14) contained the highest levels of the same-numbered fatty acids. Myristic acid was selected for modification of the fatty acid group in emulsan under conditions inhibiting fatty acid biosynthesis. The emulsifying activity, however, had no relation to the supplementation of even-numbered fatty acids (data not shown).

When myristic acid was supplemented at concentrations up to $1.0 \%$ under conditions inhibiting fatty acid biosynthesis, both the myristic acid and total fatty acid contents in emulsan increased (Fig. 3). These results suggest that myristic acid could be directly incorporated into emulsan. The emulsifying activity decreased with increasing myristic acid concentration, and decreased markedly at myristic acid concentrations above $0.4 \%$. Therefore, the myristic acid and total fatty acid contents in emulsan could be increased and the emulsifying activity could be modified within a limited range by adjusting the concentration of supplemented myristic acid under conditions inhibiting fatty acid biosynthesis.

\section{DISCUSSION}

Emulsan with a lower fatty acid content, a more hydrophilic nature, and a larger hydrodynamic volume can

TABLE 2. Fatty acid composition of emulsan produced by supplementing odd-numbered fatty acids under conditions inhibiting fatty acid biosynthesis

\begin{tabular}{|c|c|c|c|c|c|c|c|}
\hline \multirow{2}{*}{ Fatty acids in emulsan } & \multicolumn{7}{|c|}{ Fatty acid content $(\%, w / w)$} \\
\hline & Control & $\mathrm{C} 7$ & $\mathrm{C} 11^{\mathrm{a}}$ & $\mathrm{C} 13^{\mathrm{a}}$ & $\mathrm{C} 15^{\mathrm{a}}$ & $\mathrm{C} 17^{\mathrm{a}}$ & $\mathrm{C} 19^{\mathrm{a}}$ \\
\hline $\mathrm{C} 8: 0$ & 0.03 & 0.57 & 0.43 & 1.73 & 1.17 & 1.49 & 2.08 \\
\hline $\mathrm{C} 10: 0$ & $t$ & 0.18 & 0.12 & 0.22 & 0.19 & 0.20 & 0.31 \\
\hline $\mathrm{C} 12: 0$ & 0.50 & 1.13 & 1.03 & 1.37 & 0.61 & 0.75 & 1.23 \\
\hline $2 \mathrm{OH}$ & $\mathbf{t}$ & $\mathrm{t}$ & $t$ & t & $\mathrm{t}$ & t & 0.11 \\
\hline $3 \mathrm{OH}$ & $\mathrm{t}$ & 0.08 & 0.10 & 0.20 & 0.18 & 0.22 & 0.47 \\
\hline $\mathrm{C} 14: 0$ & $\mathrm{t}$ & 0.07 & 0.04 & 1 & $\mathrm{t}$ & $t$ & $\mathrm{t}$ \\
\hline $\mathrm{C} 16: 1$ & $\mathrm{t}$ & $\mathrm{t}$ & $t$ & $t$ & $\mathrm{t}$ & $\mathrm{t}$ & $\mathrm{t}$ \\
\hline $\mathrm{C} 16: 0$ & 1.27 & 1.71 & 1.53 & 0.13 & 1.02 & 1.54 & 2.18 \\
\hline C18:2 & 0.19 & $\mathrm{t}$ & $\mathrm{t}$ & $\mathrm{t}$ & $\mathrm{t}$ & $\mathrm{t}$ & $\mathrm{t}$ \\
\hline $\mathrm{C} 18: 1$ & 0.08 & $\mathrm{t}$ & $t$ & $t$ & $\mathrm{t}$ & $\mathrm{t}$ & $\mathrm{t}$ \\
\hline $\mathrm{C} 18: 0$ & 0.69 & $\mathrm{t}$ & 0.05 & 0.28 & $\mathrm{t}$ & 0.26 & 0.51 \\
\hline $\mathrm{C} 20: 0$ & 0.65 & $\mathrm{t}$ & $\mathrm{t}$ & $\mathrm{t}$ & $\mathrm{t}$ & $\mathrm{t}$ & $\mathrm{t}$ \\
\hline Odd numbered fatty acid & $\mathrm{t}$ & $\mathrm{t}$ & $\mathrm{t}$ & $t$ & $\mathrm{t}$ & $t$ & $\mathrm{t}$ \\
\hline Total fatty acid & 3.41 & 3.74 & 3.30 & 3.83 & 3.17 & 4.46 & 6.89 \\
\hline
\end{tabular}

Abbreviations are as follows: $\mathrm{C} 8: 0$, caprylic (octanoic) acid; $\mathrm{C} 10: 0$, capric (decanoic) acid; $\mathrm{C12}: 0$, lauric (dodecanoic) acid; 2-OH, 2hydroxylauric acid; 3-OH, 3-hydroxylauric acid; $\mathrm{C} 14: 0$, myristic (tetradecanoic) acid; $\mathrm{C} 16: 0$, palmitic (hexadecanoic) acid; $\mathrm{C} 16: 1$, palmitoleic acid; C18:0, stearic (octadecanoic) acid; C18:1, oleic acid; $\mathrm{C} 18: 2$, linoleic acid; and C20:0, arachidic (eicosanoic) acid.

a Fatty acid was supplemented in the methyl-ester form.

$\mathrm{t}$, Below $0.03 \%$. 
TABLE 3. Fatty acid compositions of emulsan produced by supplementing even-numbered fatty acids under conditions inhibiting fatty acid biosynthesis

\begin{tabular}{|c|c|c|c|c|c|c|c|}
\hline \multirow{2}{*}{ Fatty acids in emulsan } & \multicolumn{7}{|c|}{ Fatty acid content $(\%, w / w)$} \\
\hline & Control & $\mathrm{C} 8$ & $\mathrm{C} 10^{\mathrm{a}}$ & $\mathrm{C} 12^{\mathrm{a}}$ & $\mathrm{C} 14^{\mathrm{a}}$ & $\mathrm{C} 16^{\mathrm{a}}$ & $\mathrm{C} 18^{\mathrm{a}}$ \\
\hline $\mathrm{C} 8: 0$ & 0.03 & 1.11 & 0.45 & 0.80 & 0.09 & 1.13 & 0.72 \\
\hline $\mathrm{C} 10: 0$ & $\mathrm{t}$ & 0.35 & 0.13 & 0.29 & t & 0.55 & 0.09 \\
\hline $\mathrm{C} 12: 0$ & 0.50 & 0.98 & 0.52 & 0.95 & 0.52 & 0.95 & 1.03 \\
\hline $2 \mathrm{OH}$ & $t$ & $t$ & $t$ & $\mathrm{t}$ & t & 0.12 & $\mathrm{t}$ \\
\hline $3 \mathrm{OH}$ & $t$ & 0.71 & 0.04 & 0.48 & t & 0.93 & $t$ \\
\hline C14: 0 & $\mathrm{t}$ & 0.10 & 0.04 & 0.08 & 2.53 & 0.11 & $\mathrm{t}$ \\
\hline C16: 1 & $\mathrm{t}$ & $\mathrm{t}$ & $t$ & $t$ & $t$ & $\mathrm{t}$ & $t$ \\
\hline $\mathrm{C} 16: 0$ & 1.27 & 2.26 & 1.34 & 1.70 & 1.31 & 2.51 & 1.51 \\
\hline C18: 2 & 0.19 & $\mathrm{t}$ & $t$ & $\mathrm{t}$ & $t$ & 1. & $t$ \\
\hline C18: 1 & 0.08 & 1 & l & 1 & 1 & L & 1 \\
\hline C18:0 & 0.69 & 0.07 & 0.11 & 0.10 & 0.08 & 0.12 & 0.05 \\
\hline $\mathrm{C} 20: 0$ & 0.65 & $\mathrm{t}$ & t & $t$ & t & $\mathrm{t}$ & $\mathrm{t}$ \\
\hline Total fatty acid & 3.41 & 5.58 & 2.63 & 4.40 & 4.83 & 6.42 & 3.40 \\
\hline
\end{tabular}

Abbreviations are as follows: C8:0, caprylic (octanoic) acid; $\mathrm{C} 10: 0$, capric (decanoic) acid; $\mathrm{C} 12: 0$, lauric (dodecanoic) acid; $2-\mathrm{OH}, 2$ hydroxylauric acid; 3-OH, 3-hydroxylauric acid; C14:0, myristic (tetradecanoic) acid; C16:0, palmitic (hexadecanoic) acid; C16: 1 , palmitoleic acid; C18:0, stearic (octadecanoic) acid; C18: 1, oleic acid; C18:2, linoleic acid; and C20:0, arachidic (eicosanoic) acid.

a Fatty acid was supplemented in the methyl-ester form.

t, Below $0.03 \%$.

be obtained in culture in the presence of the antibiotic cerulenin (Table 1). In culture medium containing 3.0 $\mathrm{mg} / \mathrm{l}$ cerulenin, fatty acid biosynthesis was shown to be inhibited as was the growth of $A$. calcoaceticus (Figs. 1 and 2).

Both the composition and content of fatty acid in emulsan could be modified under conditions inhibiting fatty acid biosynthesis by the addition of various supplemental fatty acids (Tables 2 and 3). Under inhibitory conditions, the supplemental fatty acid appeared to be metabolized and biosynthesized by cells and to be incorporated into emulsan because trace amounts of various odd-numbered fatty acids were found in emulsan (Table

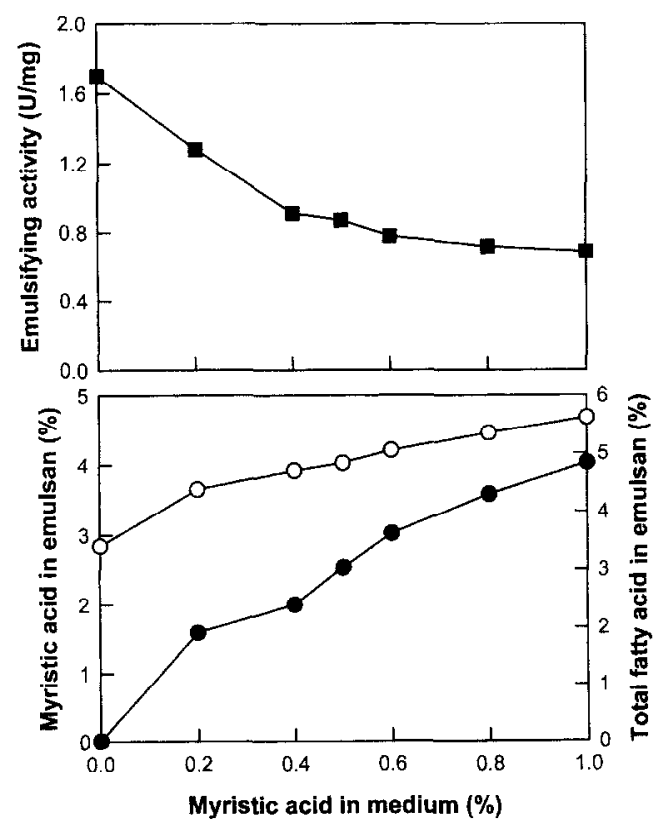

FIG. 3. Effect of the concentration of supplemented myristic acid on emulsifying activity and the contents of myristic acid and total fatty acid in emulsan under conditions inhibiting fatty acid biosynthesis. Symbols: $\square$, emulsifying activity; $\bullet$, myristic acid in emulsan; $\bigcirc$, total fatty acid in emulsan.
2). In the case of myristic acid, however, the fatty acid could be directly incorporated to emulsan because the myristic acid content in emulsan was increased by increasing the concentration of supplemental myristic acid (Fig. 3). The properties of emulsan, such as its emulsifying activity, could also be modified within a defined range by adjusting the concentration of supplemental myristic acid.

All the emulsan samples produced with odd-numbered supplemental fatty acids contained high concentrations of even-numbered fatty acids and trace amounts of oddnumbered fatty acids. These results can be cxplaincd by the fact that the odd-numbered fatty acids are degraded by $\beta$-oxidation in cells and as a result, acetyl-CoA and propionyl-CoA are predominantly produced and trace amounts of short-chain odd-numbered fatty acids are formed. Propionyl-CoA undergoes three enzymatic conversions to give succinyl-CoA which yields free succinate, an intermediate of the tricarboxylic acid cycle (17). The even-numbered fatty acids are biosynthesized from acetyl-CoA and are incorporated into emulsan and were found to be the predominant fatty acids in emulsan. The trace amounts of odd-numbered fatty acids are biosynthesized from short-chain odd-numbered fatty acids and acetyl-CoA and are incorporated into emulsan and were then found in emulsan.

There are also reports that modification of the fatty acid composition in the production of itulin (9) and mannosyl-erythritol lipids (10) affects the functional properties of each emulsifier. However, the fatty acid composition and functional properties of emulsan can be varied by changing the conditions of the $A$. calcoaceticus culture (11). For this reason, it is important to elucidate the factors influencing the fatty acid composition and functional properties of emulsan. In this study, it was found that the fatty acid composition and functional properties of emulsan such as its emulsifying activity could be modified by the use of supplemental fatty acids. 


\section{REFERENCES}

1. Fiechter, A.: Biosurfactant: moving towards industrial application. Tibtech., 10, 208-217 (1992).

2. Rosenberg, E., Zuckerberg, A., Ruvinovitu, C., and Gutnick, D. L.: Emulsifier of Acinetobacter calcoaceticus RAG-1: isolation and emulsifying properties. Appl. Environ. Microbiol., 37, 402-408 (1979).

3. Belsky, I., Gutnick, D. L., and Rosenberg, E.: Emulsifier of Acinetohacter calcoaceticus RAG-1: determination of emulsifier-bound fatty acids. FEBS Lett., 101, 175-178 (1979).

4. Rosenberg, E., Perry, A., Gibson, D. T., and Gutnick, D. L.: Emulsifier of Acinetobacter calcoaceticus RAG-1: specificity of hydrocarbon substrate. Appl. Environ. Microbiol., 37, 409-413 (1979).

5. Zuckerberg, A., Diver, A., Perry, Z., Gutnick, D. L., and Rosenberg, E.: Emulsifier of Acinetobacter calcoaceticus RAG1: chemical and physical properties. Appl. Environ. Microbiol., 37, 414-420 (1979).

6. Zosim, Z., Gutnick, D. L., and Rosenberg, E.: Properties of hydrocarbon-in-water cmulsions stabilized by Acinetobacter calcoaceticus RAG-1 emulsan. Biotechnol. Bioeng., 24, 281 (1982).

7. Yakimov, M. M., Fredrickson, H. L., and 'Tïmmis, K. N.: Effect of heterogeneity of hydrophobic moieties on surface activity of lichenysin A, a lipopeptide biosurfactant from Bacillus licheniformis BAS50. Biotechnol. Appl. Biochem., 23, 13-18 (1996).

8. Kim, S. Y., Oh, D. K., and Kim, J. H.: Biological modification of hydrophobic group in Acinetobacter calcoaceticus RAG-1 emulsan. J. Ferment. Bioeng., 84, 162-164 (1997).
9. Hourdou, M. L., Besson, F., and Michel, G.: Specific inhibition of itulin biosynthesis by cerulenin. Can. I. Microbiol., 36, 164-168 (1990).

10. Kitamoto, D., Yanagishita, H., Haraya, K., and Kitamoto, H. K.: Effect of cerulenin on the production of mannosylerythritol lipids as biosurfactants by Candida antarctica. Biotechnol. Lett., 17, 25-30 (1995).

11. Sar, N. and Rosenberg, E.: Emulsifier production by Acinetobacter calcoaceticus strains. Curr. Microbiol., 9, 309-314 (1983).

12. Shabtai, Y. and Wang, D. I. C.: Production of emulsan in a fermentation process using soybean oil (SOB) in a carbon-nitrogen coordinated feed. Biotechnol. Bioeng., 35, 753-765 (1990).

13. Greenwald, H. L., Brown, G. L., and Fineman, M. N.: Determination of the hydrophilic-lipophilic character of surface active agents and oils by a water titration. Anal. Chem., 28, 1693-1697 (1956).

14. Vance, D., Goldberg, I., Mitsuhashi, O., Bloch, K., Omura, S., and Nomura, S.: Inhibition of fatty acid synthetases by the antibiotic cerulenin. Biochem. Biophys. Res. Commun., 48, 649-656 (1972).

15. D'agnolo, G., Rosenfeld, I. S., Awaya, J., Omura, S., and Vagelos, P. R.: Inhibition of fatty acid synthesis by the antibiotic cerulenin, specific inactivation of $\beta$-ketoacyl-acyl carrier protein synthetase. Biochim. Biophys. Acta, 326, 155-166 (1973).

16. Omura, S.: The antibiotic cerulenin, a novel tool for biochemistry as an inhibitor of fatty acid synthesis. Bacteriol. Rev., 40, 681-697 (1976).

17. Lehninger, A. L., Nelson, D. L., and Cox, M. M.: Principles of biochemistry, p. 492-493. Worth Publishers, New York (1993) 